\title{
Correction to: Advances and trends on the utilization of multi-parent advanced generation intercross (MAGIC) for crop improvement
}

\author{
Kajal Samantara $\cdot$ Vincent P. Reyes $\mathbb{D} \cdot$ Nisha Agrawal $\cdot$ Sourav Ranjan Mohapatra \\ Kshirod K. Jena $(1)$
}

Published online: 19 October 2021

(C) Springer Nature B.V. 2021

\section{Correction to: Euphytica (2021) 217:189 https://doi.org/10.1007/s10681-021-02925-6}

In the above mentioned publication, Table 4 is a duplicate of Table 3 and the current Table 5 should have been labeled as Table 4 . The correct version of

Table 4 is thus published here and Table 5 is nonexcising.
The original article can be found online at https:// doi.org/10.1007/s10681-021-02925-6.

\section{K. Samantara}

Department of Genetics and Plant Breeding, Centurion University of Technology and Management,

Odisha 761211, India

\section{P. Reyes}

Graduate School of Bioagricultural Sciences, Nagoya University, Chikusa-ku, Furo-cho, Nagoya,

Aichi 464-8601, Japan

N. Agrawal

School of Biochemistry, Devi Ahilya University, Indore,

Madhya Pradesh 452001, India

\author{
S. R. Mohapatra \\ Division of Genetics and Tree Improvement, Forest \\ Research Institute, Dehradun, Uttarakhand 248006, India \\ K. K. Jena $(\bowtie)$ \\ School of Biotechnology, KIIT Deemed University, \\ Bhubaneswar, Odisha 751024, India \\ e-mail: kshirod.jena@kiitbiotech.ac.in
}


Table 4 Summary of Multi-parent Advanced Generation Intercross (MAGIC) population in various crops for other agronomic traits

\begin{tabular}{|c|c|c|c|c|c|}
\hline $\begin{array}{l}\text { Sr. } \\
\text { no }\end{array}$ & Crop & Experimental design & Trait/s identified & $\begin{array}{l}\text { Software/methods used for QTL } \\
\text { analysis }\end{array}$ & References \\
\hline 1 & $\begin{array}{l}\text { Oat (Avena } \\
\text { sativa) }\end{array}$ & $\begin{array}{l}\text { Eight spring oats crossed in } \\
\text { halfdiallel with } 42 \text { funnels } \\
\text { AIORIL }\end{array}$ & $\begin{array}{l}\text { Heading dates and } \\
\text { plant maturity } \\
\text { dates, the height } \\
\text { of the plant, yield }\end{array}$ & QTL, LMC, GWAS & $\begin{array}{l}\text { Huang } \\
\text { et al. } \\
\text { (2015) }\end{array}$ \\
\hline 2 & $\begin{array}{l}\text { Rice (Oryza } \\
\text { sativa } \mathrm{L} .)\end{array}$ & $\begin{array}{l}\text { A global MAGIC population made } \\
\text { through intercrossing of } \\
\text { eightway F1 indica and eight- } \\
\text { way F1 japonica founders which } \\
\text { resulted in a 150, 16-way crosses } \\
\text { advanced for S8 (selfing } \\
\text { generation) }\end{array}$ & $\begin{array}{l}\text { Yield and grain } \\
\text { quality } \\
\text { parameters }\end{array}$ & $\begin{array}{l}\text { GWAS, Multi-parent interval } \\
\text { mapping, Bayesian genomic } \\
\text { networking, RAP DB, QTARO, } \\
\text { and GRAMENE databases for } \\
\text { Candidate QTLs }\end{array}$ & $\begin{array}{l}\text { Zaw et al. } \\
\text { (2019) }\end{array}$ \\
\hline 3 & $\begin{array}{l}\text { Rice (Oryza } \\
\text { sativa L.) }\end{array}$ & $\begin{array}{l}\text { Eight indica founder parents were } \\
\text { crossed to generate } 1328 \text { S7 lines }\end{array}$ & $\begin{array}{l}\text { Grain quality } \\
\text { (amylose content, } \\
\text { grain length, } \\
\text { gelatinization } \\
\text { temperature) }\end{array}$ & TASSEL for GWAS & $\begin{array}{l}\text { Bandillo } \\
\text { et al. } \\
(2013)\end{array}$ \\
\hline 4 & $\begin{array}{l}\text { Tomato } \\
\text { (Solanum } \\
\text { lycopersicum } \\
\text { L.) }\end{array}$ & $\begin{array}{l}\text { Four normal tomatoes and four } \\
\text { cherry tomatoes were selected as } \\
\text { founders and have produced } 397 \\
\text { RILs from four generations of } \\
\text { crosses and three selfings }\end{array}$ & Weight of fruit & R/mpMap & $\begin{array}{l}\text { Pascual } \\
\text { et al. } \\
\text { (2014) }\end{array}$ \\
\hline 5 & $\begin{array}{l}\text { Strawberry } \\
\text { (Fragaria } x \\
\text { ananassa })\end{array}$ & $\begin{array}{l}\text { Six founder lines were intermated } \\
\text { to generate } 1060 \text { intercross } \\
\text { population }\end{array}$ & $\begin{array}{l}\text { Traits related to } \\
\text { Fruit quality }\end{array}$ & - & $\begin{array}{l}\text { Wada } \\
\text { et al. } \\
(2017)\end{array}$ \\
\hline 6 & $\begin{array}{l}\text { Cotton } \\
\text { (Gossypium } \\
\text { herbaceum) }\end{array}$ & $\begin{array}{l}\text { Eleven diversified varieties were } \\
\text { intermated up to F5 and then } \\
\text { selfed by SSD to generate } 550 \\
\text { RILS }\end{array}$ & Quality of fiber & GAPIT, TASSEL & $\begin{array}{l}\text { Islam et al. } \\
\text { (2016), } \\
\text { Li et al. } \\
\text { (2016) }\end{array}$ \\
\hline
\end{tabular}

Publisher's Note Springer Nature remains neutral with regard to jurisdictional claims in published maps and institutional affiliations. 